\title{
Long-period modelling of MEDNET waveforms for the December 13, 1990 Eastern Sicily earthquake
}

\author{
Domenico Giardini $\left({ }^{1}\right)$, Barbara Palombo $\left({ }^{2}\right)$ and Nicola Alessandro Pino $\left({ }^{2}\right)$ \\ $\left.{ }^{1}\right)$ Dipartimento di Scienze Geologiche, III Università degli Studi, Roma, Italy \\ $\left.{ }^{2}\right)$ Istituto Nazionale di Geofisica, Roma, Italy
}

\begin{abstract}
The availability of broad-band digital data allows the analysis of the seismic signal in the low-noise frequency band for structural and seismic source studies. We model complete seismograms - surface and body waves for the December 13, 1990, Eastern Sicily earthquake, recorded at regional distances on the MEDNET stations model-independent strategy to fit complete seismoment tensor is carried out following two approaches: a) a phase velocity curves stable and consistent rosults: the path to fit surface waves in the 40-100 s range. Both methodologies yield dyne.cm dyetic seismograms ang to magnitude values of $m_{b}=5.5, M_{S}=5.7, M_{W}=M_{L}=5.8$. In a second stage, syn«minors» integration are generated by full reflectivity in the $5-50 \mathrm{~s}$ period range, with a new code based on the rive average velocity models for the Central waves in the 30-50 s range and body waves to $5 \mathrm{~s}$ periods, we de1990 Eastern Sicily earthquake to be in the 13-17 km depth range.
\end{abstract}

Key words seismic moment - inversion techniques very-broad-band waveforms - regional earthquakes - Eastern Sicily

\section{Introduction}

Among the outstanding seismological issues is the accurate, rapid monitoring of regional seismicity (100-3000 $\mathrm{km}$ distance range).

The need to develop accurate methods for the rapid evaluation of size and geometry of the seismic source is crucial in the densely inhabited Mediterranean basin, where even a shock of moderate proportions may cause a catastrophe. The December 13, 1990, Eastern Sicily earthquake caused widespread damage
$(I=$ VII-VIII Mercally maximum intensity $)$ and 19 casualties. Several estimates of the size of this event have been issued: magnitudes $m_{b}=5.5$ and $M_{S}=5.3$ (NEIC), $M_{D}=5.6$ (TRI), $M_{D}=5.3$ (TTG), $M_{D}=5.2$ (THE), $M_{D}=5.1$ and $M_{L}=5.4$ (ING) (it should be noted that this spread is all too frequent, reflecting the true limitation of the magnitude scales); seismic moments $M_{0}=4 \times 10^{24}$ dyne $\cdot \mathrm{cm}$ (PPT) and $M_{0}=3.3 \times 10^{24}$ dyne $\cdot \mathrm{cm}(\mathrm{CMT})$. In the immediate aftermath of the event the only available size estimate was a preliminary $M_{D}=4.9$ issued by ING, since the rapid determination procedures of EMSC and NEIC were not triggered. Consistent estimates of the focal geometry were derived only later using arrivals 
from teleseismic stations (Amato et al., personal comunication, 1991; De Rubeis et al., personal comunication, 1991) and by waveform modelling (CMT).

Modelling digital waveforms is the main tool of modern seismology for structural and seismic source studies. Waveform modelling at teleseismic distances $(>3000 \mathrm{~km})$ is relatively straightforward; body waves travel mostly in the more homogeneous lower mantle and surface waves average out the structural heterogeneity along very long paths; the effect of the seismic source in the seismogram is dominant and the synthesis of complete low-frequency seismograms can be efficiently carried out with accurate algorithms using depth-dependent velocity models.

Modelling regional waveforms (100-3000 $\mathrm{km}$ distance range) is a more complex exercise, due to theoretical and practical limitations. At this distance the contributions of the source complexity and of the propagation in the heterogeneous lithosphere and upper mantle are equally important. The availability of digital

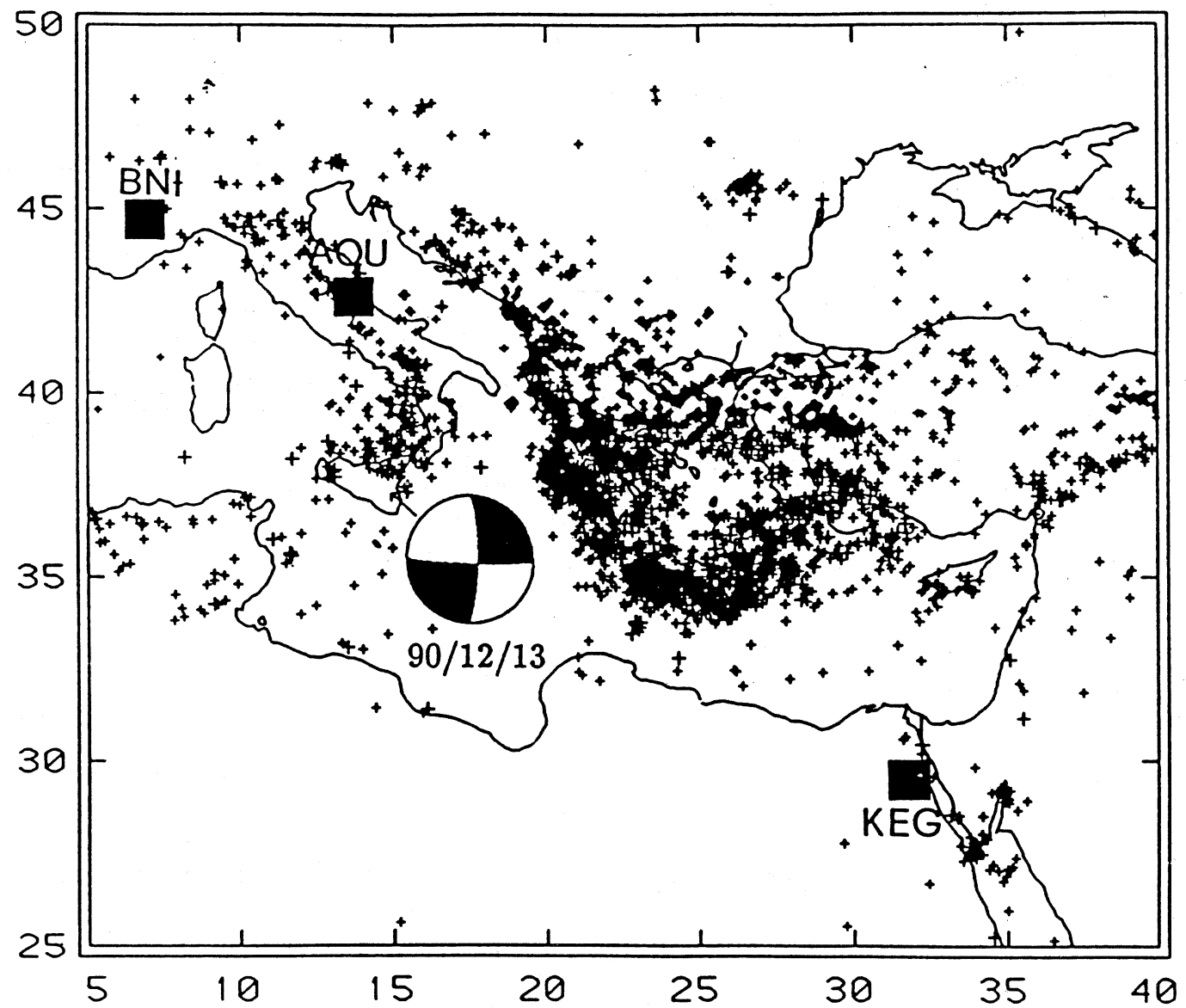

Fig. 1. Seismicity of the Central-Eastern Mediterranean region, from the ISC 1964-1990 catalogue; a minimum threshold of $m_{b}=4$ is applied. Also shown is the distribution of the MEDNET stations which recorded the December 13, 1990, Eastern Sicily earthquake (squares) and the location and fault plane mechanism of the event. 
waveforms collected at regional distances has always been limited, due to the sparse distribution of global digital stations; in addition, regional seismic phases arrive very close to each other and often simultaneously in the seismogram, posing serious problems of identification and separation.

Today the installation of regional seismic networks in many areas of the world provides digital data of unprecedented quality at regional scale and seismology is renewing efforts to study the seismic process of regional earthquakes (Dreger and Helmberger, 1990; Karabulut et al., 1991; Ritsema et al., 1991; Nakanishi et al., 1992). Here we model complete seismograms for the December 13, 1990, Eastern Sicily earthquake, recorded at regional distances on the MEDNET stations AQU, BNI and KEG.

Our aim is to invert for the moment tensor in different frequency bands and to assess the stability and the precision of the results with regard to the structural model and the inversion procedures used. Part of the material included here and more details on the technical procedures can be found in Giardini (1991) and Giardini et al. (1993a,b). A second goal is to model surface waves at periods of 30-50 s and body waves in the 5-50 s range for source and structural investigations.

\section{The MedNet data}

MEDNET is the very-broad-band seismographic network installed by the Istituto Nazionale di Geofisica of Roma in countries of the Mediterranean area, in the framework of the WorldLab program Plato-I; at the expected completion in 1994, MEDNET will monitor the regional seismicity with 15 sites (Boschi et al., 1991). The Eastern Sicily event provides a good test for the rapid moment tensor determination using MEDNET data; three stations were operating on that day (BNI, KEG, AQU; fig. 1).

The high standards of VBB technology (Wielandt and Streckeisen, 1986; Wielandt and Steim, 1986) and of the MEDNET installation procedures (Giardini et al., 1992) allow Earth

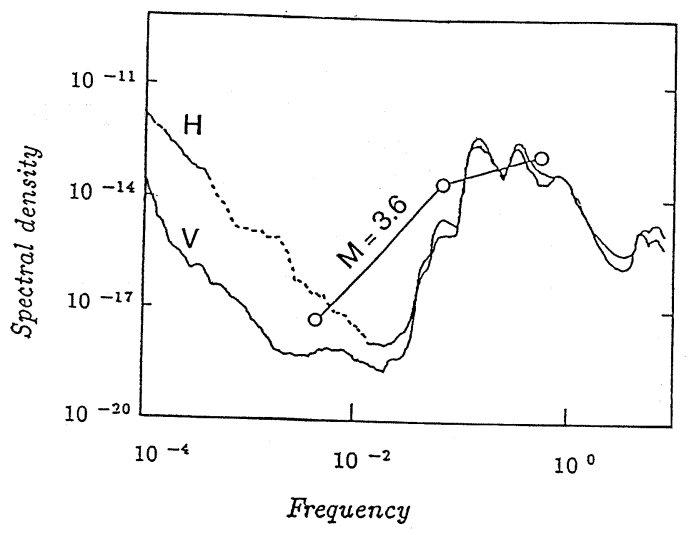

Fig. 2. Experimental curves of minimum Earth noise averaged over the vertical (V) and horizontal (H) components of MEDNET stations (in power spectral density units; after Mazza and Morelli, 1992). Superimposed is the acceleration expected from a $M_{S}=3.6$ earthquake at $2000 \mathrm{~km}$ distance.

noise in the low-frequency band and wave trains to be recorded with excellent signal-tonoise ratios at regional distances for small earthquakes ( $m=4-5$; Wielandt and Steim, 1986); the best performance in the long-period band is obtained in the $40-130 \mathrm{~s}$ period range (fig. 2).

The extended dynamic range $(140 \mathrm{~dB})$ of VBB instrumentation makes it possible to record scale large local events $(m=6$ at less than $50 \mathrm{~km}$ distance). The possibility of telemetering waveforms for several MEDNET sites allows automatic procedures for data scanning and processing to be implemented with minimal intervention of the operator for a rapid evaluation of the seismic activity.

Figure 3 displays the vertical record at AQU on the VBB and LP channels, and the LP trace filtered in different frequency bands.

\section{Modelling regional waveforms}

Waveform modelling must be adapted to the period range and epicentral distance of interest. Several approaches are possible to model seis- 


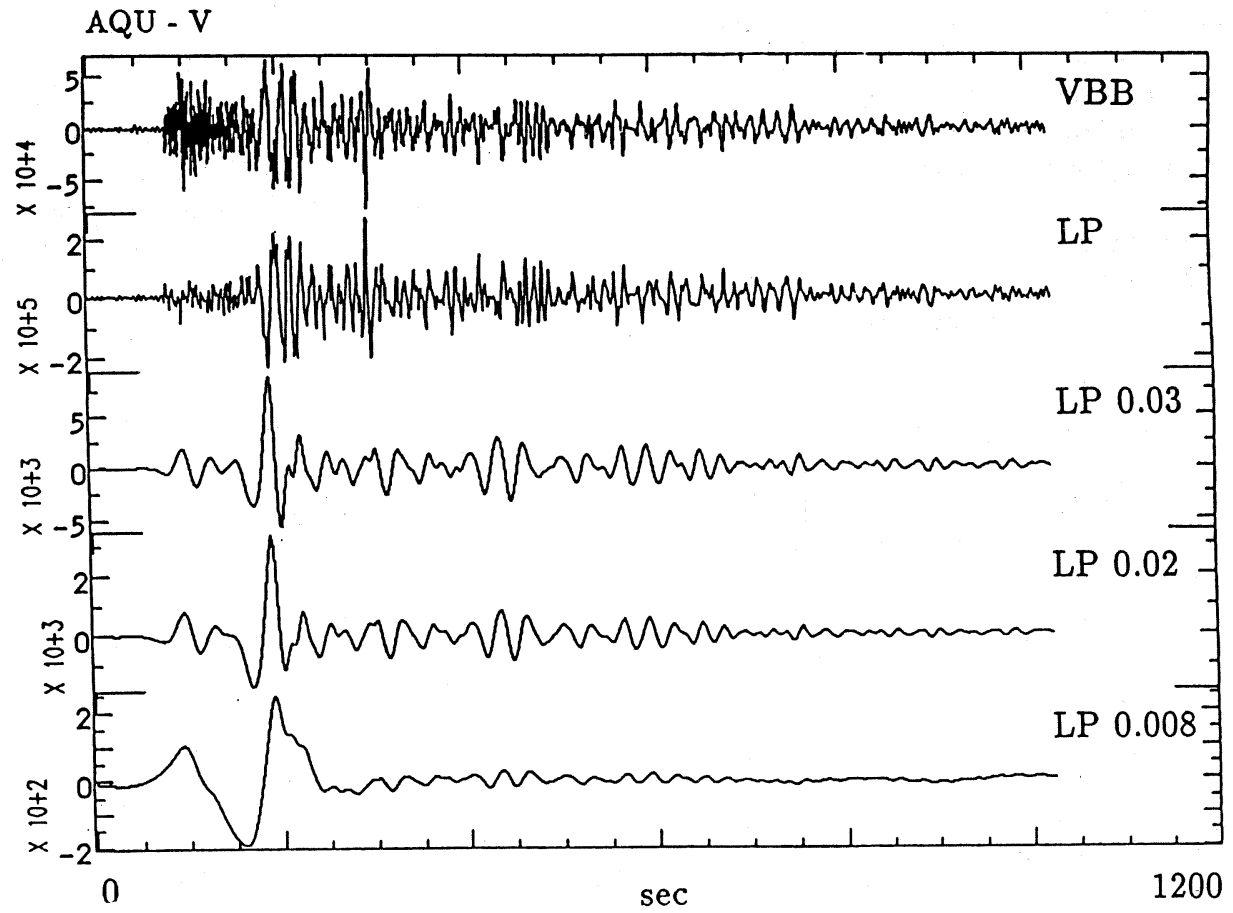

Fig. 3. Vertical trace of the December 13, 1990, Eastern Sicily earthquake recorded at AQU on the VBB and LP channels, and the LP trace low-passed filtered at different frequencies: 30, 20 and $8 \mathrm{mHz}$. The amplitude scale is in digital counts.

mograms recorded at regional and teleseismic distances:

- seismograms at very long period (more than $100 \mathrm{~s}$ ) are dominated by surface waves for shallow earthquakes, and are easily modelled using a standard Earth model; complete seismograms can be synthetized by normal mode summation at regional and teleseismic epicentral distances;

- in the long-period 40-100 s range the dispersion of surface waves depends strongly on the lithospheric structure and requires the calibration of phase velocity curves along each path, or phase velocity maps for whole regions, a task which, on a global scale, can be attained with sufficient precision only in a few regions; although lithospheric models of the EuropeanMediterranean region have been derived from the existing data (Panza et al., 1980; Calcagnile et al., 1982), an accurate three-dimensional structural model capable to reproduce all path heterogeneities in the Mediterranean region over the wide frequency band of interest is still wanting; body waves are a significant component of the seismogram, especially the $S$ phase and its reflections, and can be modelled using an average velocity model;

- at periods of 10-40 s the amplitude of surface waves is influenced by the focussingdefocussing effects caused by the laterally heterogeneous velocity structure and by lateral variations in $Q$; surface wave modelling requires sophisticated algorithms dealing with 2-D and 3-D structures; the synthesis of body waves also requires the knowledge of the structural model, but it can be accomplished using depth-dependent velocity models, pro- 
vided the path is not crossing a steeply dipping interface (subduction areas) or a major structural interface (plate boundaries);

- at periods of $1-10 \mathrm{~s}$ the aim is to model individual body wave phases.

Here we show examples of the four types to recover source properties for the December 13, 1990, Eastern Sicily earthquake.

\section{Long-period moment tensor inversion: 130-100 s}

The inversion for the moment tensor of regional earthquakes over a wide range of dimensions $\left(10^{23}-20^{28}\right.$ dyne $\left.\cdot \mathrm{cm}\right)$ requires the synthesis of accurate regional seismograms over a wide range of frequencies. Our first approach is to constrain the analysis to the longperiod (100-130 s) portion of the low-noise spectral window. Indeed, body- and surfacewaves at periods exceeding $100 \mathrm{~s}$ are insensitive to regional-scale heterogeneities in seismic velocity and can be modelled using an average structural model. Further advantages of longperiod modelling are the simplicity of the parametrization of the seismic source, taken as a point source, and the availability of efficient codes for the generation of accurate synthetic seismograms, valid also in the near field, by normal mode summation (Woodhouse, 1988) using radially symmetric Earth models (PREM; Dziewonski and Anderson, 1981).

We use an algorithm suitable for the determination of moment tensors of regional earthquakes (Giardini et al., 1993a,b). The main elements of the code are:

- we invert waveforms in the time domain, band-passed in the $8-10 \mathrm{mHz}$ frequency band; for smaller events we choose data windows characterized by higher signal-to-noise ratio;

- a library of complete synthetic seismograms is built, computed at fixed epicentral distances for selected hypocentral depths, by normal modes summation (Woodhouse, 1988) using the PREM model (Dziewonski and Anderson, 1981);

- we invert three-components, 20-min rec- ords, containing long-period body waves and the first orbit of Rayleigh and Love waves;

- a time shift is computed for each trace, accounting for the source half-duration, for the centroid mislocation of large earthquakes and for any phase contamination due to a strongly heterogeneous path; the frequency range (8-10 $\mathrm{mHz}$ ) is nearly monochromatic and warrants the use of a single, frequency-independent correction term;

- the source process is parametrized with the full moment tensor and the added constraint of null volumetric component;

- depth is constrained by variance minimization on inversions at different trial depths, following Romanowicz and Suarez (1983);

- for large earthquakes we use data in narrow frequency bands with periods up to 500$1000 \mathrm{~s}$.

Waveform fitting for the Eastern Sicily event is shown in fig. 4; we obtain a seismic moment $M_{0}=3.7 \times 10^{24}$ dyne $\cdot \mathrm{cm}$, in agreement with the published CMT solution and corresponding to magnitude values of $m_{b}=5.5, M_{S}$ $=5.7, M_{W}=M_{L}=5.8$ (using regression laws from Heaton et al., 1986).

We perform several tests to assess the stability of the solution. In fig. 5 we show the focal mechanisms obtained using the joint MEDNET data and in single station inversions; for comparison the CMT mechanism and two fault planes solutions (S1, Amato et al., personal comunication, 1991; S2, De Rubeis et al., personal comunication, 1991) are also displayed. For all the solutions we list the seismic moment and the variance to the whole MEDNET dataset (defined as the ratio between the misfit and the data norm and expressed in percentiles). The agreement obtained by inversion of single station data is very encouraging, indicating that even a single VBB station permits reliable control of the seismic source of regional earthquakes (cfr. Ekström et al., 1986).

As we invert waves in an almost monochromatic frequency band, our depth resolution is not ideal; indeed the most important consideration is again the stability of the solution, in terms of dimension and fault geometry, also in the presence of erroneous depth. Figure 6 

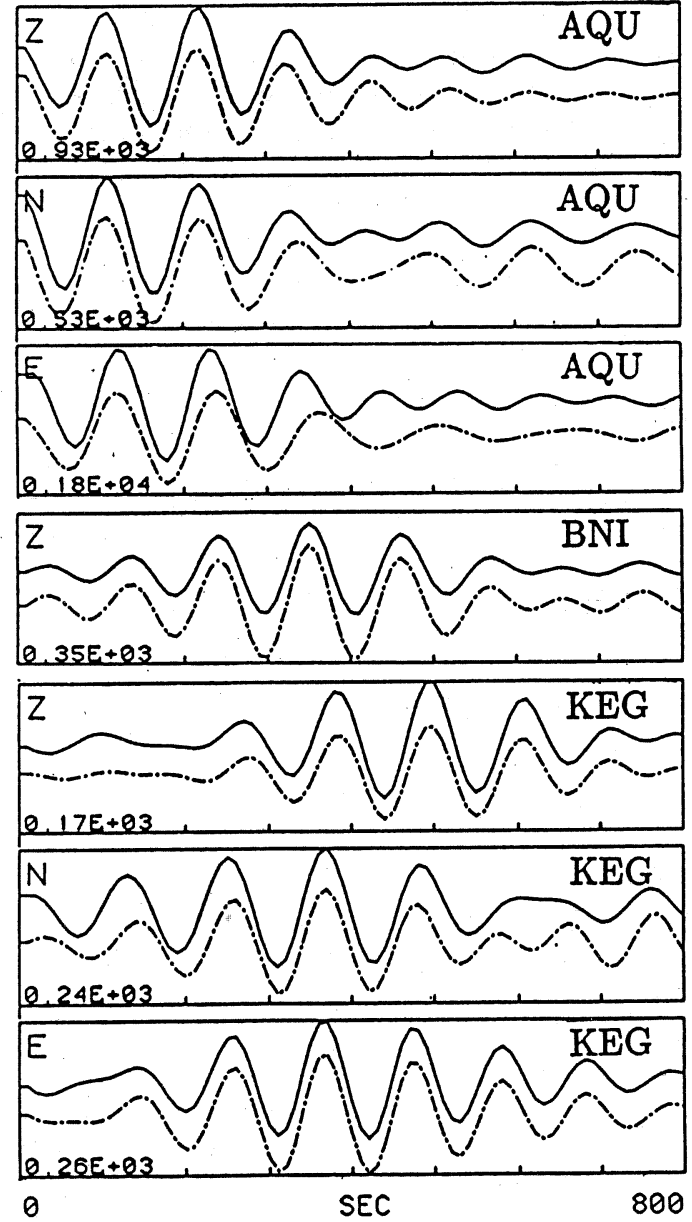

Fig. 4. Waveform modelling for the December 13, 1990, Syracuse event. For each component we show $20 \mathrm{~min}$ of long-period seismograms, band-passed in the $8-10 \mathrm{mHz}$ frequency bands; data are indicated by continuous lines, synthetic seismograms by dashed lines.

shows the depth dependence of our solution; we display the normalized variance, the focal mechanism and the seismic moment obtained at different trial depths. The variance curve with depth shows the Eastern Sicily hypocenter to be rather deep for a crustal earthquake; the acceptable depth range is $15-30 \mathrm{~km}$, with seismic moment ranging between 3.7 and $3.9 \times 10^{24}$ dyne $\cdot \mathrm{cm}$.
In addition, we may estimate the uncertainty associated with the single independent elements of the moment tensor. A larger instability is usually associated with the $M_{r \theta}$ and $M_{r \phi}$ components of the moment tensor, which can be reduced by applying a weak minimization scheme in the inversion; however, the value of the seismic moment and the dip-slip component of the moment tensors can be affected by the minimization scheme. A test for the Eastern Sicily event (fig. 7) shows that the moment tensor solution is stable unless a very strong constraint on the moment size is applied. Solutions with a wide range of minimization parameters $(\varepsilon=0.0-0.05)$ have very similar moment tensor elements; the $M_{r \theta}$ and $M_{r \phi}$ components of the moment tensor are always rather small, as the moment tensor is dominated by the strike-slip $M_{\theta \phi}$ component; the seismic moment for these four inversions decreases from 4.0 to $3.7 \times 10^{24}$ dyne $\cdot \mathrm{cm}$, while the normalized variance remains in the 0.043-0.045 range. Only with a very strong constraint $(\varepsilon=1.0)$ does the solution degrade considerably (variance $=.13$ ) and the seismic moment diminish to $2.5 \times 10^{24}$ dyne.cm. As the moment tensor in all cases is dominated by the strike-slip components, its geometry remains stable.

\section{Long-period moment tensor inversion: 100-40 s}

A second approach focusses on the inversion of surface waves in the $40-100 \mathrm{~s}$ period range, adopting a regionalized, frequency dependent phase-velocity model to account for the 3-D lithospheric structure. This method cannot be extended to higher frequencies, as the focussing and defocussing due to lateral heterogeneities in velocity and the laterally varying $Q$ induce large amplitude effects on surface waves, requiring $2-\mathrm{D}$ and $3-\mathrm{D}$ modelling.

Our approach to invert regional waveforms is based on the consideration that (a) although lithospheric models of the European-Mediterranean region have been derived from the existing data (ex. Panza et al., 1980), an accurate three-dimensional structural model capable of 

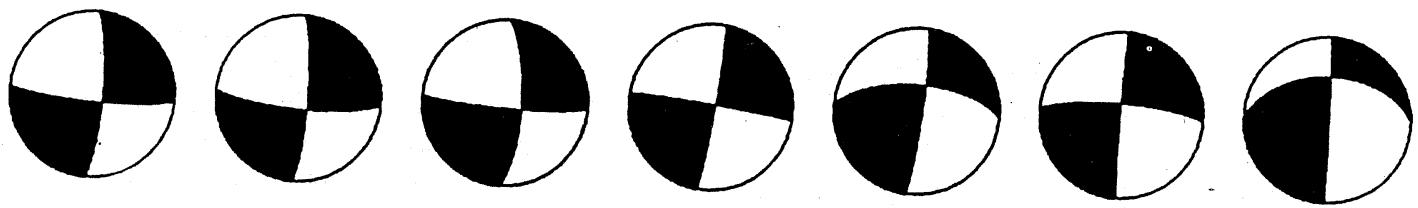

\begin{tabular}{|c||c|c|c||c||c|c|}
\hline MedNet & AQU & BNI & KEG & CMT & S1 & S2 \\
\hline $.37 / 4$ & $.39 / 4$ & $.23 / 1$ & $.30 / 4$ & $.33 / 18$ & $-/ 5$ & $-/ 9$ \\
\hline
\end{tabular}

Fig. 5. Focal mechanisms obtained using the joint MEDNET data and in single station inversions (AQU, BNI, KEG); for comparison also the CMT mechanism and two fault planes solutions (S1, Amato et al., personal comunication, 1991; S2, De Rubeis et al., personal comunication, 1991) are displayed. For all the solutions we list the seismic moment (in units of $10^{25}$ dyne. $\mathrm{cm}$ ) and the normalized variance (the ratio between the misfit and the data norm, expressed in percentiles) to the whole MEDNET dataset.

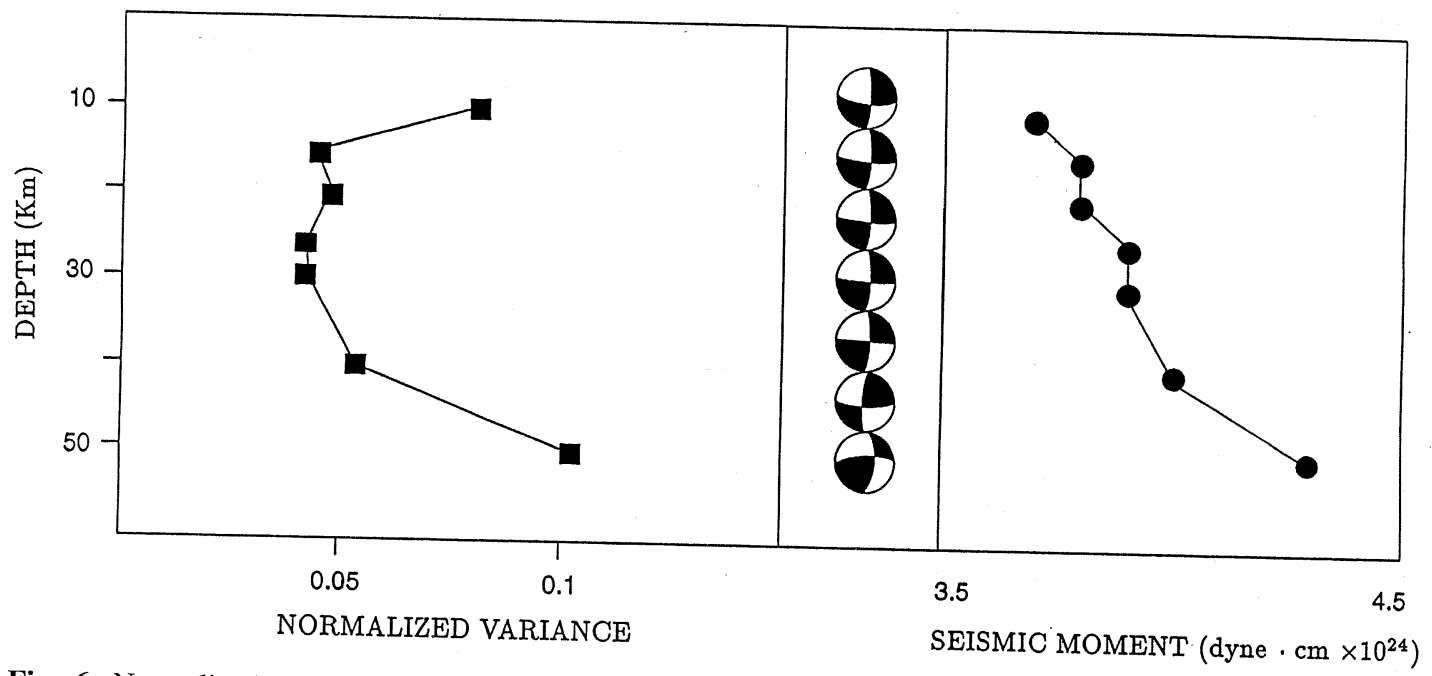

Fig. 6. Normalized variance, focal mechanism and seismic moment obtained in inversions at different trial
depths.

reproducing all path heterogeneities in the Mediterranean region over the wide frequency band of interest is still wanting, (b) a global average model (ex. PREM) can reproduce the amplitude of surface waves down to periods of $30-40$ s quite accurately, and (c) what is required is thus a simple strategy to calibrate the phase velocity along each path in the $40-100 \mathrm{~s}$ period range.

The main elements of our approach are:
- to use the initial reference library of synthetic seismograms computed at fixed epicentral distances for selected hypocentral depths for the 1-D model (PREM);

- to compute phase correction curves for Rayleigh and Love waves in the 40-100 s band by smoothing phase differences between data and synthetics on specific station-event paths for moderate-large earthquakes, using moment tensor solutions derived at low frequency; 


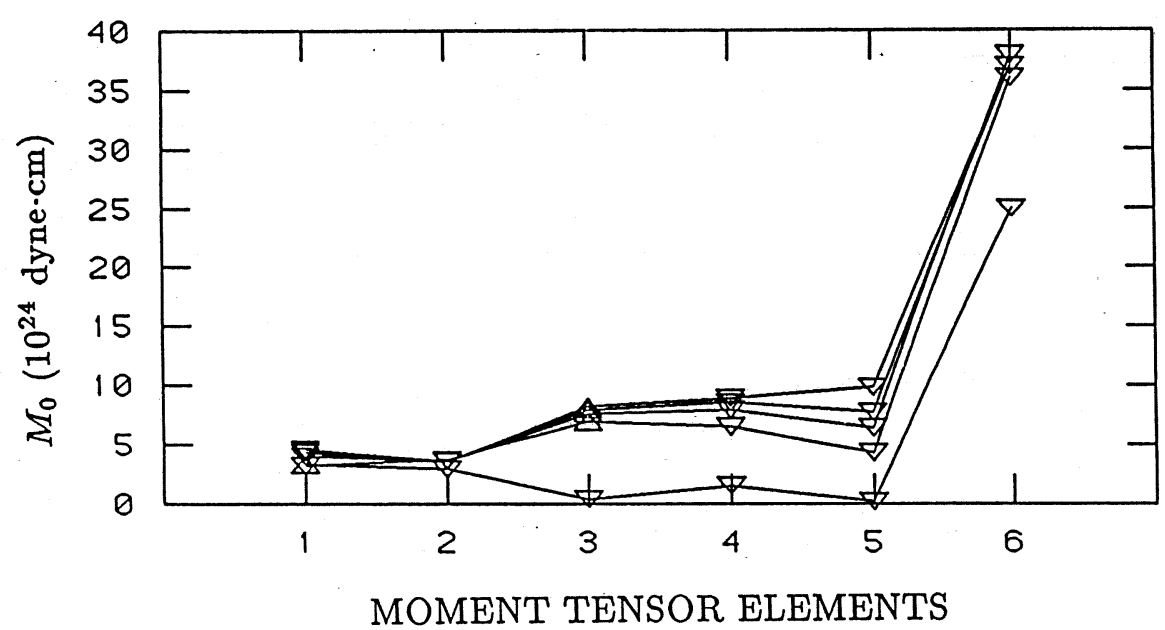

Fig. 7. Absolute amplitude of the six elements of the moment tensor for five inversions performed with different minimization constraints $(\varepsilon=0.0,0.01,0.02,0.05,1.0)$. Upward triangles indicate positive values; downward triangles indicate negative ones. The seismic moments obtained in the five inversions are respectively $4.0,3.9,3.9,3.7,2.5 \times 10^{24}$ dyne $\cdot \mathrm{cm}$; the corresponding normalized variances are $0.043,0.043,0.043$, 0.045 and 0.125 .

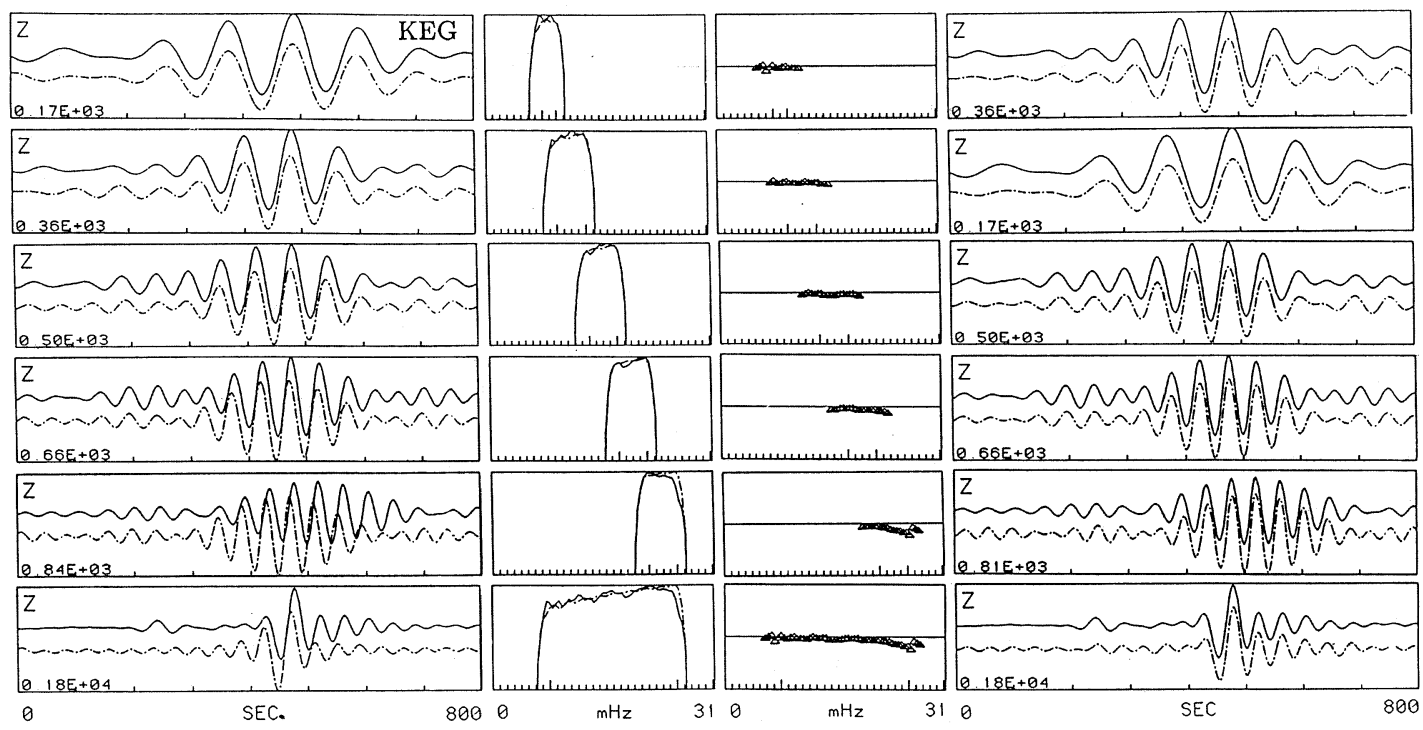

Fig. 8. Multiple-frequency forward modelling for the vertical component of station KEG. To the left are the comparisons of data and synthetics computed for the reference model in five narrow frequency bands (from top to bottom $8-10,10-14,14-18,18-22,22-26$ and $8-26 \mathrm{mHz}$ ) and in the whole $8-26 \mathrm{mHz}$ band; the panels in the middle show the corresponding amplitude spectra for data and synthetics (the vertical logarithmic scale spans three orders of amplitude) and the phase difference between the complex spectra of data and synthetics (on a scale of $\pm 4 \pi$ ); to the right is the fit obtained by correcting the path with the smoothed phase difference curve. 


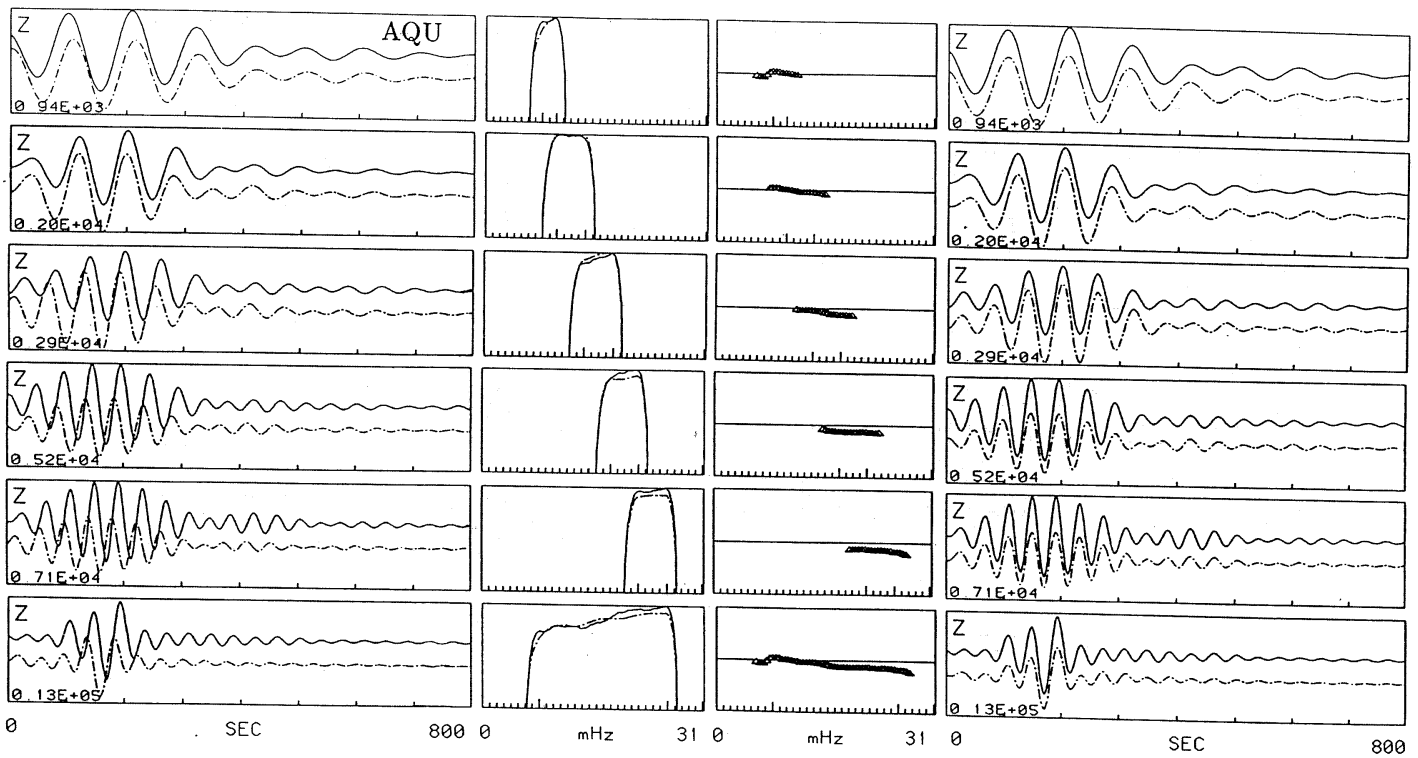
Fig. 9. Multiple-frequency forward modelling for the vertical component of station AQU. See caption of
fig. 8 for explanations.

- to build a library of phase correction curves for calibrated paths;

- to invert for the moment tensor in multiple frequency bands by combining the libraries of synthetic seismograms and of phase correction curves, following the approach outlined above.

Figure 8 displays examples of multiple-frequency, forward modelling for the vertical component of station KEG. To the left are the comparisons of data and synthetics computed for the reference model in five narrow frequency bands and in the whole $8-26 \mathrm{mHz}$ band; the panels in the middle show the corresponding amplitude spectra for data and synthetics and the phase difference between the complex spectra; to the right are the fit obtained by correcting the path with the smoothed phase difference curve. Figure 9 shows the same for the vertical component of station AQU. The amplitude fit is very good across the whole frequency band, although the moment tensor used was computed in the narrow $8-10 \mathrm{mHz}$ band shown in the top display (the slight amplitude discrepancy visible at high frequency for $A Q U$ is due to faulty instrumental calibration). When phase corrections are introduced, the alignment and the fit between data and synthetics in time domain is good across the frequency band of interest.

Figure 10 shows examples of moment tensor inversions using MEDNET waveforms filtered in narrow $(2 \mathrm{mHz}$ ) frequency bands, from 9 to $25 \mathrm{mHz}$, and in the whole $8-26 \mathrm{mHz}$ range (cfr. figs. 8 and 9). The agreement among the fault geometries and the seismic moment values is quite encouraging, considering the very limited number of traces available for the inversion and the adoption of coarse phase calibrations.

\section{Broad-band modelling: 5-50 s}

Recently some experiments with broad-band regional data (Dreger and Helmberger, 1990; Zielhuis and Nolet, 1991) have shown that the use of stratified models allows the main characteristics of seismograms to be reproduced at local and regional distance, for relatively simple tectonic environments. Here we verify the 

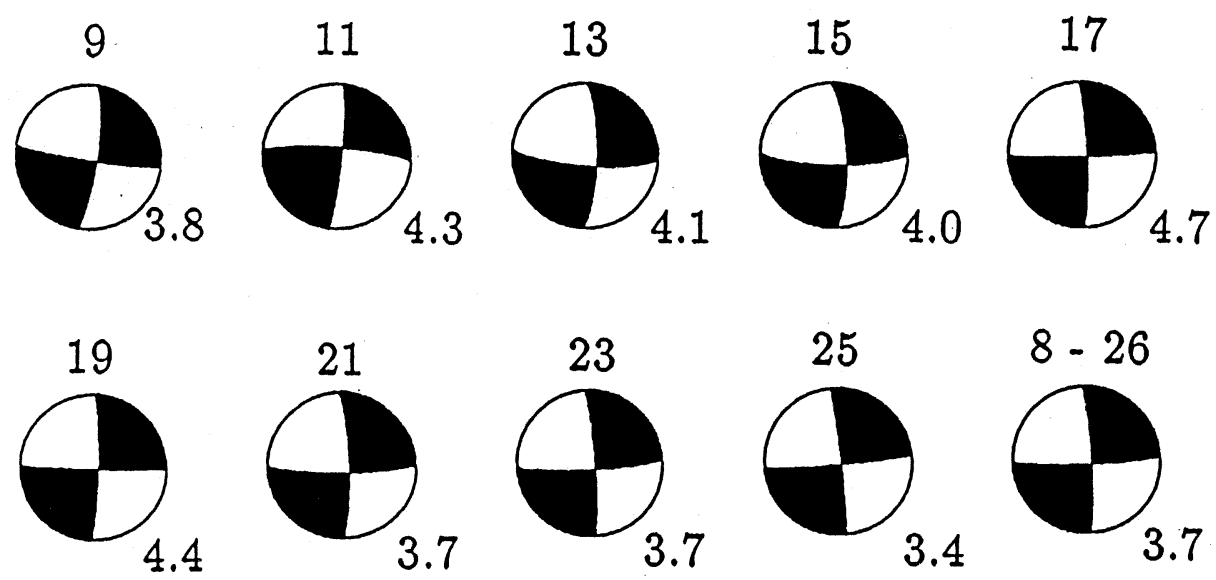

Fig. 10. Moment tensor solutions from inversions in narrow ( $2 \mathrm{mHz}$ ) frequency bands, from 9 to $25 \mathrm{mHz}$, and in the whole $8-26 \mathrm{mHz}$ range. For each solution we show the best double-couple mechanism and the seismic moment, in units of $10^{24}$ dyne $\cdot \mathrm{cm}$.

limits of application of stratified models for structural and source analyses at regional scale.

Several considerations can be made. The use of a stratified model to fit regional waveforms implies averaging the heterogeneities along the source-station path; this approximation adapts better to surface waves, which for their nature sample with a low frequency spectral content all the shallow structures along the source-station path. Body waves instead are sensitive to the superficial structure near the source and the station, while travelling mostly at depth along the rest of the path. It is thus likely that, especially at high frequency, a model representing a good average structure for surface waves will not reproduce the body waves adequately; a typical example is the case of hypocenter and station located on continental margins and the path crossing through an oceanic basin.

A second consideration regards the need to make use of body and surface waves on a wide band of frequencies. Indeed, the problem of obtaining a structural model from monochromatic surface waves is highly controversial; the use of surface waves spanning a large frequency range adds structural resolution with depth, while modelling body waves allows to constrain the hypocentral depth, the impedance layering near the surface, the depth of the main deep interfaces, and a mean deep structure.

The investigation of the lithospheric structure through inversion techniques has been attempted, although these methods are applicable only in the case of very simple structural models (at most one layer over a halfspace) and generally for small epicentral distance; they are not applicable to model a complete seismogram at regional distance in the case of a multi-layered structural model.

Here we employ a trial-and-error procedure, constraining the initial model with known structural elements along the path and adjusting the model to reproduce the observed characteristics of the signal: the relative amplitude of the surface and body waves, the complexity of the body waves, the duration of the surface waves coda, the arrival times of the $P, S$ and surface wave phases. Once a good average model is found, we reproduce separately the fine structure of body waves phases at higher frequencies to calibrate the depth and impedance of the main interfaces, remaining close to the mean model.

Since we use a point source representation of the source, we position our analysis in the flat portion of the displacement source spec- 
trum. The high-frequency limit of our analysis is thus connected to the source duration; for a moderate earthquake like the Eastern Sicily one, $\left(M_{0}=3.7 \times 10^{24}\right.$ dyne $\left.\cdot \mathrm{cm}\right)$, the expected duration of the fault is of few seconds and we model waves in the 5-50 period range.

Complete synthetic seismograms can be computed efficiently for a layered structure following the modal or the reflectivity approach. The reflectivity method has been widely used in the literature (Fuchs and Muller, 1971; Kennett, 1983). Here (Appendix A) we develop an algorithm to model body waves $(P-S V, S H)$ and surface waves, based on the full reflectivity approach, following the formulation of Woodhouse (1980).

While reflectivity algorithms are now readily available, we preferred to conduct our own development, as these codes require extensive testing; for example, for the definition of the step and range for the integration in frequency and wave number. Comparative tests against published algorithms allowed the verification of the numerical accuracy of our procedure; a significant difference emerged in the comparison with published synthetics and was confirmed by the author to be due to errors on his side (Kennett, personal comunication).

The advantages of adopting an analytic solution lie in the rapidity of calculus, in the accuracy and completeness of the synthetics, in the explicit integration over the plane $\omega-k$, making possible the a priori choice of the frequencies and the branch to model. The use of depth-dependent velocity distribution limits the modelling to relatively simple structural environments. Dispersion and attenuation are introduced by choosing elastic parameters with an imaginary component (Woodhouse, 1980; Kennett, 1983). We choose the stations AQU and KEG to apply the proposed approach for different epicentral distances and tectonic paths.

$K E G$ - The geophysical framework of the South-Eastern Mediterranean from Sicily to Africa is characterized by the collision between the African and Eurasian plates, with the transition from the Ionian Sea to the African craton (Cloetingh et al., 1979; Calcagnile et al., 1982) and the subduction under the Tyrrhenian and Aegean Seas (Spakman, 1989).

In fig. 11 we show the comparison between data and synthetics for the vertical $(A, B)$ and radial $(\mathrm{C}, \mathrm{D})$ components at station $\mathrm{KEG}$, filtered at $50(\mathrm{~A}, \mathrm{C})$ and $30(\mathrm{~B}, \mathrm{D}) \mathrm{s}$. As antici-
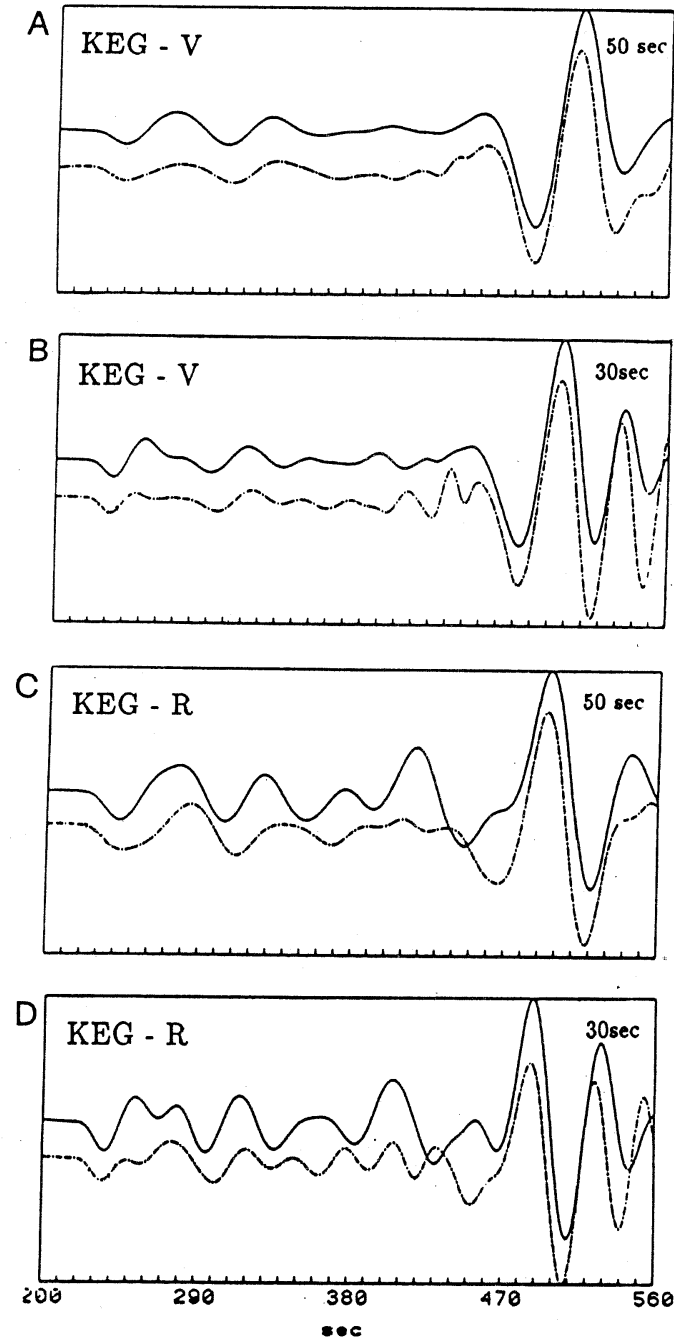

Fig. 11. Waveform fitting for the vertical $(A, B)$ and radial $(\mathrm{C}, \mathrm{D})$ components at station $\mathrm{KEG}$, filtered at $50(\mathrm{~A}, \mathrm{C})$ and $30(\mathrm{~B}, \mathrm{D})$ s. Continuous lines indicate data, dashed lines are used for synthetics; the time scale is in seconds from the origin time. 
pated, the complete seismograms are dominated by the Rayleigh wave train. Seismic moment values derived from forward modelling at different frequencies are stable $\left(3.4-3.7 \times 10^{24}\right.$ dyne. $\mathrm{cm}$ ) and comparable with the value obtained by long period analysis (Giardini, 1991).

Figure 12 displays the family of structural models $\left(v_{P}, v_{S}, \rho\right)$, which reproduce the waveforms along the KEG path; the dashed area provides a quantitative indication of the model uncertainty and of the structural heterogeneity along the path.

$A Q U$ - The Syracuse - L'Aquila, $600 \mathrm{~km}$ path crosses such different tectonic provinces as the volcanic region of Etna, the Tyrrhenian subduction zone (Giardini and Velonà, 1991), the Moho climb in the Tyrrhenian basin (Steinmetz et al., 1983) and the Appenninic crust (Mostardini and Merlini, 1986).

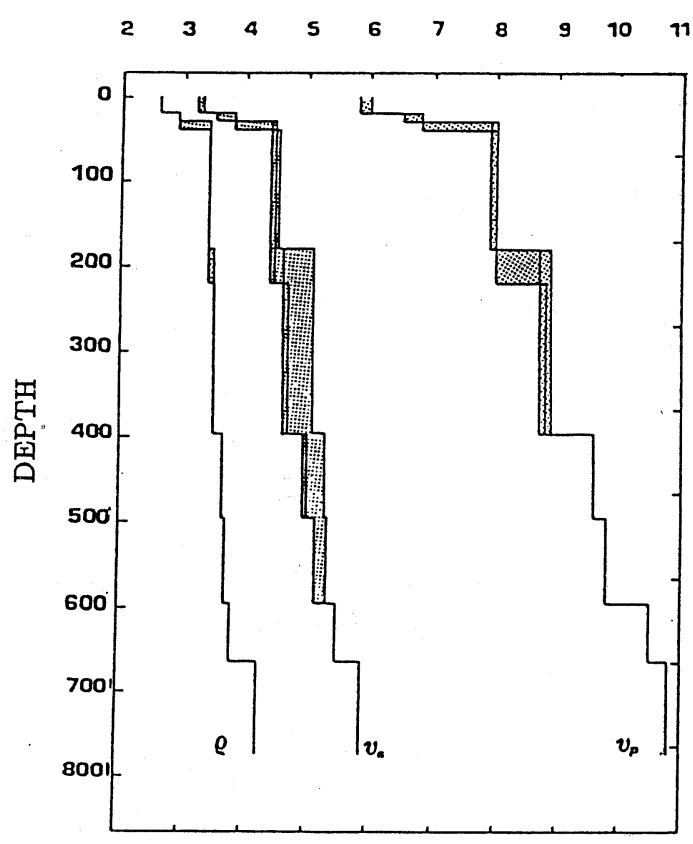

Fig. 12. Structural models used to reproduce the waveforms along the KEG path (see fig. 11), in terms of seismic velocities $v_{P}$ and $v_{S}$ (in $\mathrm{km} / \mathrm{s}$ ) and density $\rho$ (in $\mathrm{gr} / \mathrm{cm}^{3}$ ).
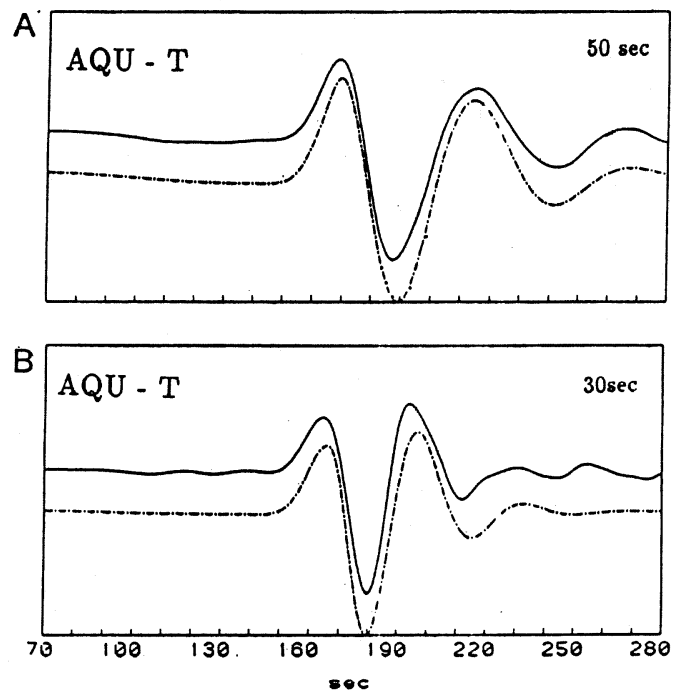

Fig. 13. Waveform fitting for the transversal component at station AQU, filtered at 50 (A) and 30 (B) s. Continuous lines indicate data, dashed lines are used for synthetics; the time scale is in seconds from the origin time.

To constrain the initial model we make use of information on the crustal and lithospheric structure from studies of gravimetry (Giese and Morelli, 1975), Moho depth and $P_{n}$ velocity for Italian earthquakes (Mele and Valensise, 1987), DSS and active seismology (Scarascia, 1982; Ferrucci et al., 1989; Nicolich, 1989), OBS observations (Steinmetz et al., 1983) and surface wave dispersion (Panza et al., 1980; Calcagnile et al., 1982; Cassinis, 1983). Even along this complex structure the assumption of a stratified model produces good results in the $30-50 \mathrm{~s}$ range. Figure 13 shows the comparison between data and synthetics for the transversal component at AQU, filtered at 50 (A) and 30 (B) $\mathrm{s}$ and dominated by the Love waves.

Modelling the body waves at higher frequency allows the depth of the event and the local structure in the source and station areas to be constrained. Figure 14 compares data and synthetics computed for different trial depths between 11 and $21 \mathrm{~km}$ and low-passed filtered at $10 \mathrm{~s}$. The best fit is achieved for the hypocenter in the $13-15 \mathrm{~km}$ depth range. 


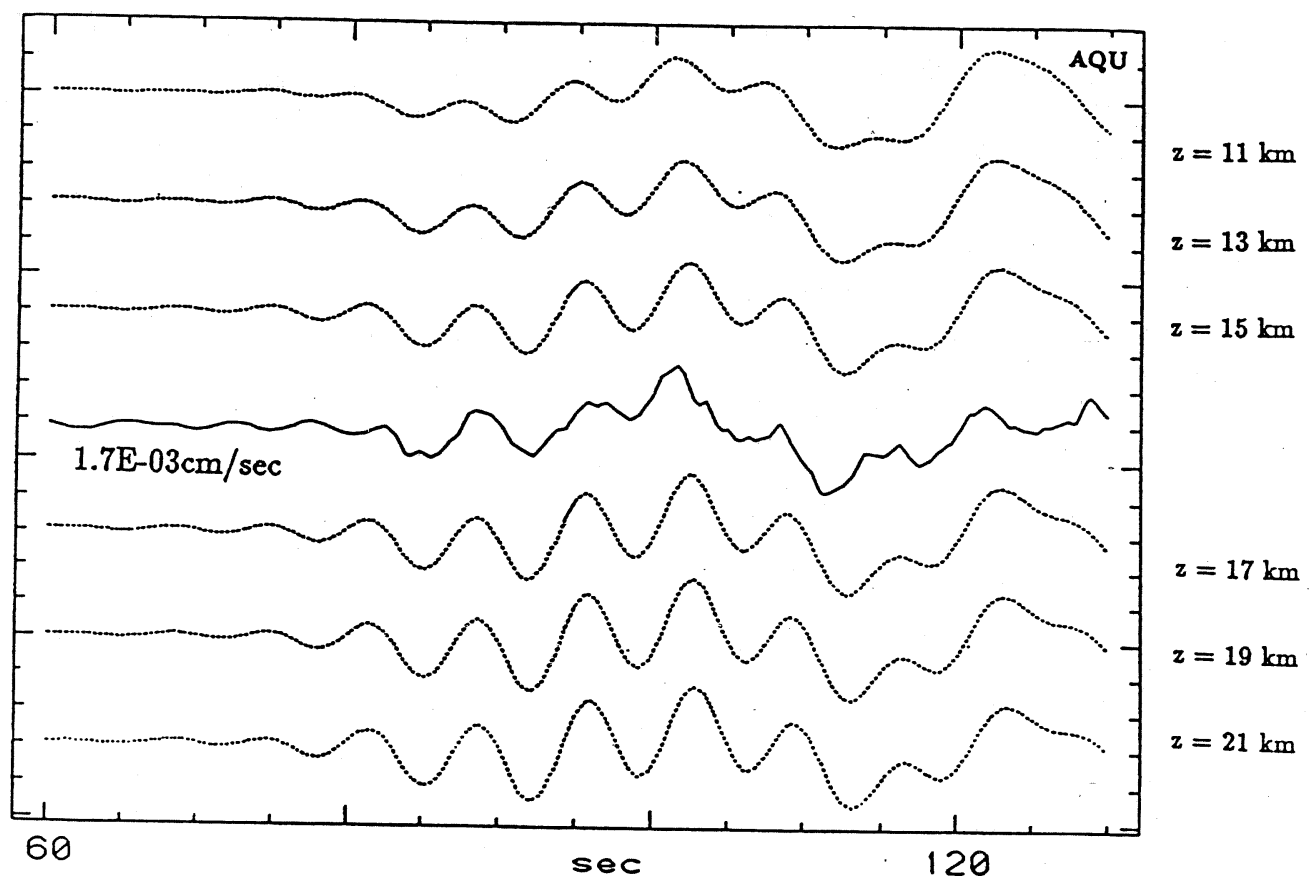

Fig. 14. Comparison between data (thick line) and synthetics computed for different trial depths between 11 and $21 \mathrm{~km}$ and low-passed filtered at $10 \mathrm{~s}$; normalized variances (the ratio between the misfit and the data norm) obtained at the six trial depths are $0.44,0.28,0.35,0.49,0.61,0.73$, constraining the hypocenter in the $13-15 \mathrm{~km}$ range.

\section{Conclusions}

The availability of broad-band digital data allows the analysis of the seismic signal in the low-noise frequency band for structural and seismic source studies.

We model complete seismograms - surface and body waves - for the December 13, 1990, Eastern Sicily earthquake, recorded at regional distances on the MEDNET stations AQU, BNI and KEG.

The inversion for the moment tensor is carried out following two approaches: we use a model-independent strategy to fit complete seismograms in the $100-130 \mathrm{~s}$ period range and we calibrate phase velocity curves along each path and invert surface waves in the 40-100 s range. Both methodologies yield stable and consistent results. The December 13, 1990, Eastern Sicily earthquake had a seismic mo- ment of $M_{0}=3.7 \times 10^{24}$ dyne $\cdot \mathrm{cm}$, corresponding to magnitude values of $m_{b}=5.5, M_{S}=5.7$, $M_{W}=M_{L}=5.8$.

We then model surface waves in the $30-50 \mathrm{~s}$ range and body waves to $5 \mathrm{~s}$ period, with a new full reflectivity code based on the «minors» integration, deriving average velocity models for the Central and Eastern Mediterranean and constraining the hypocenter of the 1990 Eastern Sicily earthquake to be in the 13$17 \mathrm{~km}$ depth range.

\section{Acknowledgements}

D.G. is grateful to Prof. J.H. Woodhouse for the collaboration in writing and testing the reflectivity code for the seismogram synthesis and for initiating him to the art of handling the Earth's normal modes, and to Profs. A.M. 
Dziewonski and J.H. Woodhouse for the experience gained on the CMT method while at Harvard University.

We thank Profs. H. Kanamori and B. Romanowicz for useful insight and comments. The MedNet Data Center is commended for collecting and promptly making available to the seismological community digital data of excellent quality. We acknowledge the leading role of Prof. Enzo Boschi and of the WorldLab in the deployment of MEDNET and in supporting this work.

\section{REFERENCES}

Boschi, E., D. Giardini and A. Morelli (1991): MedNET: the broad-band seismic network for the Mediterranean, Il Cigno Galileo Galilei, Roma.

Calcagnile, G., F. D'Ingeo, P. Farrugia and G.F. PANZA (1982): The lithosphere in the Central Eastern Mediterranean Area, Pure Appl. Geophys., 120, 389406.

CASsinis, S. (1983): Seismicity and crustal structure in the Italian region: a preliminary zoning, Boll. Geofis. Teor. Appl., 25, 3-26.

Cloetingh, S., G. Nolet and R. Wortel (1979): On the use of Rayleigh wavegroup velocities for the analysis of continental margins, Tectonophysics, 59, 335-346.

Dreger, D.S. and D. Helmberger (1990): Broad-band modelling of local earthquakes, Bull. Seismol. Soc. Am., 80, 1162-1179.

DzIEWOnSKI, A.M. and D.L. ANDERson (1981): Preliminary Reference Earth Model (PREM), Phys. Earth Planet. Inter., 25, 297-356.

Dziewonski, A.M., T.A. Chou and J.H. WoOdhouse (1981): Determination of earthquake source parameters from waveform data for studies of global and regional seismicity, J. Geophys. Res., 86, 2825-2852.

Ekströom, G., A.M. DzIEWONSKI and J.M. STEIM (1986): Single station CMT: application to the Michoacan, Mexico, earthquake of September 19, 1985, Geophys. Res. Lett., 13, 173-176.

Ferrucci, F., G. Gaudiosi, N.A. Pino, G. Luongo, A. Hirn and L. Mirabile (1989): Seismic detection of a major Moho upheaval beneath the Campania volcanic area (Naples, Southern Italy), Geophys. Res. Lett., 16, 1317-1320.

Fuchs, K. and G. Muller (1971): Computation of synthetic seismograms with the reflectivity method and comparison with observations, Geophys. R. Astron. Soc., 23, 417-433.

GiARdini, D. (1991): Analisi di dati MEdNET per il terremoto della Sicilia Orientale del 13 Dicembre 1990: (1) modellazione a lungo periodo, in Contributi allo Studio del Terremoto della Sicilia Orientale del 13 Dicembre 1990, edited E. BoschI and A. BAsILI, Publication of the Istituto Nazionale di Geofisica, Roma, n. 537, 103-114.
Giardini, D. and M. Velonà (1991): Deep seismicity of the Tyrrhenian Sea, Terra Nova, 3, 57-64.

Giardini, D., E. Boschi, S. Mazza, A. Morelli, D. Ben Sari, D. NaJid, H. Benhallou, M. Bezzeghoud, H. Trabelsi, M. Hafaied, R. Kebeasy and E. Ibrahim (1992): Very-broad-band seismology in Northern Africa under the MEDNET project, Tectonophysics, 209, 17-30.

Giardini, D., E. Boschi and B. Palombo (1993a): Moment tensor inversion from MEDNET data: (2) earthquakes of the Mediterranean, Geophys. Res. Lett., 20, 273-276.

Giardini, D., B. Palombo and E. Boschi (1993b): The determination of earthquake size and source geometry in the Mediterranean Sea, in Recent Evolution and Seismicity of the Mediterranean Area, edited by E. Boschi et al., (Kluwer), 213-238.

Giese, P. and C. Morelli (1975): Crustal structure of Italy. Structural model of Italy, Quaderni della Ricerca Scientifica, 90, 453-489.

GilberT, F. and G.E. BACKus (1966): Propagator matrices in elastic wave and vibration problems, Geophysics, 31, 326-332.

Heaton, T., F. Tajima and A.W. Mori (1986): Estimating ground motion using recorded accelerograms, Surveys in Geophysics, 8, 25-83.

Hudson, J.A. (1969): A quantitative evaluation of seismic signals at teleseismic distances. I - Radiation from a point source, Geophys. J. R. Astron. Soc., 18, 233-249.

Karabulut, H., T.M. Boyd and S. SiPKIN (1991): Determination of earthquake source parameters from regional waveforms, Eos (Trans. Amer. Geophys. Un.), 72, 304.

Kennett, B.L.N. (1983): Seismic Wave Propagation in Stratified Media (Cambridge University Press, Cambridge).

MAZZA, S. and A. Morelli (1992): Background seismic noise from MEDNET very-broad-band stations, in Proceedings and Activity report 1988-1990, XXII General Assembly of the European Seismological Commission, vol. 1, 197-202.

Mele, F.M. and G. VAlensise (1987): Un modello crostale per la localizzazione di eventi sismici regionali rilevati dalla rete sismica nazionale centralizzata dell'ING, in Proceedings, $6^{\circ}$ Annual Meeting GNGTS, 1377-1395.

Mostardini, F. and S. Merlini (1986): Sezioni geologiche e proposte di modello strutturale, Appennino Centro-Meridionale, in Proceedings, $73^{\circ}$ Assembly SGI, 177-202.

NAKAnishi, I., T. Moriya and M. Endo (1992): The November 13, 1990 earthquake off the coast of the Primorskij region, the Eastern Russia, Geophys. Res. Lett., 19, 549-552.

NiCOLICH, R. (1989): Crustal structures from seismic studies in the frame of the European Geotraverse (Southern Segment) and Crop Projects, in The Litosphere in Italy, Advances in Earth Science Research (Accademia Nazionale dei Lincei), 41-62.

Panza, G., S. Mueller and G. Calcagnile (1980): The gross features of the litosphere-astenosphere system in Europe from seismic surface waves and body waves, Pure Appl. Geophys., 118, 1209-1213. 
Ritsema, J., J. Zhang, K. Kuge and T. LaY (1991): Stability of time domain waveform inversion for regional earthquake source parameters, Eos (Trans. Amer. Geophys. Un.), 72, 304.

ROMANOWICZ, B. and G. SuAREZ (1983): On an improved method to obtain the moment tensor and depth of earthquakes from the amplitude spectrum of Rayleigh waves, Bull. Seismol. Soc. Am., 73, 1513-1526.

SCARASCIA, S. (1982): Crustal structure in the Southern Appennines region from D.S.S. data (1980 experiments), Proceedings, EGS-ESC Assembly.

SPAKMAN, W. (1989): Tomographic images of the upper mantle below Central Europe and Mediterranean, Terra nova, 2, 542-553.

Steinmetz, L., F. Ferrucci, A. Hirn, C. Morelli and R. NiCOLICH (1983): A $550 \mathrm{~km}$ long Moho traverse in the Tyrrhenian Sea from OBS recorded $P_{n}$ waves, Geophys. Res. Lett., 10, 428-431.

TAKEUCHI, H. and M. SAITO (1972): Seismic surface waves, in Methods of Computational Physics (Academic Press, New York), vol. 11.

Wielandt, E. and G. Streckeisen (1982): The leafspring seismometer: design and performance, Bull. Seismol. Soc. Am., 72, 2349-2367.

WIELANDT, E. and J.M. STEIM (1986): A digital verybroad-band seismograph, Annales Geophysicae, 4B3, 227-232.

WoOdHouse, J.H. (1980): Efficient and stable methods for performing seismic calculations in stratified media, in Physics of the Earth Interior, Proc. Int. School of Physics «E. Fermi» (North-Holland), vol. 78, 127-151.

Woodhouse, J.H. (1988): The calculation of eigenfrequencies and eigenfunctions of the free oscillations of the Earth and the Sun, in Seismological Algorithms, edited by D.J. Doornbos (Academic Press), 321370.

Zielhuis, A. and G. Nolet (1991): A 3-D $S$ velocity model for Europe obtained with partitioned waveform tomography, EOS (Trans. Amer. Geoph. Un., 72, 349.

Appendix A. The reflectivity method.

The response of a horizontally stratified medium to a point source is obtained by integration over frequency $(\omega)$ and wave number ( $\kappa)$ (Hudson, 1969). If the medium is isotropic, the response is conveniently separated in the $P-S V$ and $S H$ components. In a polar cylindrical coordinate system $(z, r, \varphi)$, a point source at depth $z=z_{S}$ produces an elastic displacement field:

$$
\boldsymbol{u}=\boldsymbol{u}(z, r, \varphi)=\int_{-\infty}^{\infty} d \omega e^{i \omega t} \sum_{m=-\infty}^{\infty} \frac{1}{2 \pi} \int_{0}^{\infty} k d k\left\{b_{1}^{S} \boldsymbol{R}_{k}^{m}+b_{2}^{S} \boldsymbol{S}_{k}^{m}+b_{1}^{T} \boldsymbol{T}_{k}^{m}\right\}
$$

where $\boldsymbol{R}_{k}^{m}, \boldsymbol{S}_{k}^{m}$ and $\boldsymbol{T}_{k}^{m}$ are spherical harmonic functions (Takeuchi and Saito, 1972).

The coefficients $b_{1}^{S}(z, k, m, \omega)$ and $b_{2}^{S}(z, k, m, \omega)$ are elements of the four components stress-displacement vector $\boldsymbol{b}^{S}$, linked to the components of motion $P-S V$; similarly, $b_{1}^{T}(z, k, m, \omega)$ is an element of the two components stress-displacement vector $\boldsymbol{b}^{T}$, linked to the component of motion $S H$. The two vectors, for $k, m$ and $\omega$ fixed, are solutions of the system of differential equations

$$
\begin{cases}\partial_{z} \boldsymbol{b}^{S}=\omega A^{S} \boldsymbol{b}^{S} & \left(z_{a}<z<z_{s}, z_{s}<z<z_{b}\right) \\ \partial_{z} \boldsymbol{b}^{T}=\omega A^{T} \boldsymbol{b}^{T} & \left(z_{a}<z<z_{s}, z_{s}<z<z_{b}\right)\end{cases}
$$

The matrices $A^{S}$ and $A^{T}$ are defined for each homogeneous layer of the depth-dependent structural model. Boundary conditions in $z_{a}$ and $z_{b}$ will depend on the specific problem; generally, every solution for $\boldsymbol{b}^{S}$ will be a linear combination of two elementary vectors which, in the case of free surface in $z_{a}$, impose a condition of null traction, or, at the bottom of the structure for $z_{b}$, correspond to $P-S V$ waves propagating in the half-space below. The vectors $\boldsymbol{b}^{S}$ and $\boldsymbol{b}^{T}$ admit only one discontinuity in correspondence of the seismic source

$$
\left[\boldsymbol{b}^{S}\right]_{z_{s}-0}^{z_{s}+0}=\boldsymbol{s}^{S}\left[\boldsymbol{b}^{T}\right]_{z_{s}-0}^{z_{s}+0}=\boldsymbol{s}^{T}
$$


where $s^{S}$ and $s^{T}$ can be written as functions of the elements of the tensor moment $M(t)$ (Hudson, 1969) and the spectrum of the source is defined as

$$
\bar{M}(\omega)=\frac{1}{2 \pi} \int_{0}^{\infty} M(t) e^{-i \omega t} d t
$$

The analytical solution of the differential equation system (A.1) for the components $P-S V$ is given by the propagator $P\left(z, z_{0}\right)$ :

$$
P\left(z, z_{0}\right)=\exp \left[\omega A^{S}\left(z-z_{0}\right)\right]
$$

with the initial condition $\boldsymbol{P}\left(z_{0}, z_{0}\right)=\boldsymbol{I}$. Physically the propagator describes the stress-displacement vector $\boldsymbol{b}^{S}(z)$ in any point of the medium when $\boldsymbol{b}^{S}\left(z_{0}\right)$ is known:

$$
\boldsymbol{b}^{S}(z)=P\left(z, z_{0}\right) \boldsymbol{b}^{S}\left(z_{0}\right)
$$

A similar solution can be obtained for the $S H$ component. Woodhouse (1980) develops the full expansion for the $P-S V$ field; we derive here the $\mathrm{SH}$ formulation.

To apply the boundary conditions, $\boldsymbol{b}^{S}$ and $\boldsymbol{b}^{T}$ are propagated from the source in $z_{s}$ to the free surface in $z_{a}$ and to the limit with the halfspace below in $z_{b}$, by successive multiplication of the propagators corresponding to the different layers in the structure

$$
\boldsymbol{b}^{S}\left(z_{a}\right)=P\left(z_{a}, z_{1}\right) P\left(z_{1}, z_{2}\right) \ldots \ldots P\left(z_{N-1}, z_{s}\right) \boldsymbol{b}^{S}\left(z_{s}\right)
$$

The progressive application of the propagator can lead to loss of numerical precision when modelling high frequency waves in a multi-strata structure; to avoid this problem we follow the scheme proposed by Gilbert and Backus (1966) and developed by Woodhouse (1980). For any two solutions of the system, $\boldsymbol{b}_{1}^{S}$ and $\boldsymbol{b}_{2}^{S}$, the vector of the six anti-symmetric products of their elements

$$
\left[b_{1 i}^{S} b_{2 j}^{S}-b_{1 j}^{S} b_{2 i}^{S}\right]_{i \neq j}
$$

defined minor, satisfies a sixth-order differential equation system, derived from the system for $\boldsymbol{b}^{S}$; the propagation of minors avoids numerical instabilities and loss of precision. 